\title{
The Performance of SLNR Beamformers in Multi-User MIMO Systems
}

\author{
Khalid W. Hameed ${ }^{1,2}$, Ahmed M Abdulkhaleq ${ }^{1,2}$, Yasir Al-Yasir ${ }^{1}$, \\ Naser Ojaroudi Parchin ${ }^{1}$, Ashwain Rayit ${ }^{3}$, Majid Al Khambashi ${ }^{4}$, Raed A. Abd- \\ Alhameed $^{1}$ and James M. Noras ${ }^{1}$ \\ ${ }^{1}$ Bradford University, Bradford BD7 1DP, UK \\ ${ }^{2}$ Al-Nahrain University /Iraq \\ ${ }^{3}$ SARAS Technology Limited, Leeds LS12 4NQ, UK \\ ${ }^{4} \mathrm{Al}-$ Zahra College for Women, Oman
}

\begin{abstract}
Beamforming in multi-user MIMO (MU-MIMO) systems is a vital part of modern wireless communication systems. Researchers looking for best operational performance normally optimize the problem and then solve for best weight solutions. The weight optimization problem contains variables in numerator and dominator: this leads to so-called variable coupling, making the problem hard to solve. Formulating the optimization in terms of the signal to leakage and noise ratio (SLNR) helps in decoupling the problem variables. In this paper we study the performance of the SLNR with variable numbers of users and handset antennas. The results show that there is an optimum and the capacity curve is a concave over these two parameters. The performances of two further variations of this method are also considered.
\end{abstract}

Keywords: Beamforming, Generalized Eigenvalue decomposition, MU-MIMO, Optimal point, SLNR.

\section{Introduction}

There are steadily increasing demands for higher data rates and channel capacity, with MIMO systems a strong possible solution for higher capacity without increased power transmission. MIMO includes SISO, MISO and SIMO configurations, with variations such as point-to-point [1], multi-user (MU-MIMO) [2] and network or multi-cell MIMO [3].

Though MU-MIMO resembles point-to-point transmission in depending on the state of the channel to transmit signals, it differs in the decoding procedure, with users usually assumed to be non-cooperating. MU-MIMO also depends on the diversity between users to achieve multiplexing between transmissions to users sharing the same time-frequency resource. This is achieved by precoding, also called beamforming.

The simplest beamforming strategy is the zero forcing (ZF) or channel inversion method [4]. This basic method suffers from poor performance at high noise figures, 
and can be enhanced using regularized ZF [5], sometimes called MMSE [6]; however, some dimensional constraints need to be satisfied, such that the total number of receiving antennas should be less than or equal to the number of antennas at the base station. This condition limits the system geometry. Another approach is to optimize the weights of the beamformer to improve performance. The optimization is either to reduce the total transmitted power [7], the power per antenna [8] or to increase the capacity [9], while keeping other parameters constant. This is done by framing the solving optimization problem in terms of the signal to interference and noise ratio (SINR). This approach has the drawback of coupled variables between different users: an increase in signal power level for one user will increase the leakage (interference) for other users. Another promising optimization technique proposed in [10] and later developed in [11] and [12] uses the signal to leakage and noise ratio (SLNR).

This paper examines the performance of the SLNR ratio under variation of SNR, the relation between base station antenna, number of users and antenna per user, developing previous work $[13,14]$. Results show that there are some limitations to be considered during the design of a system. The performance of the system is not necessarily monotonic; it can exhibit a peak depending on the number of base station antennas, number of users and number of antennas per user.

\section{System Mathematical Model}

Consider a cell that contains a single base station with $\mathrm{M}$ antennas, transmitting signals to $\mathrm{K}$ users each with $\mathrm{N}$ antennas as shown in Figure 1.

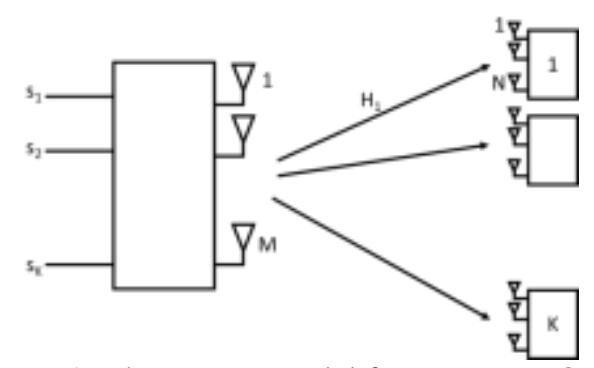

Figure 1: The system model for MU-MIMO.

The base station uses the same time-frequency resource to send data to these users. The channel from the base station to user $\mathrm{i}$ is given by:

$$
H_{i}=\left[\begin{array}{ccc}
h_{1,1, i} & \cdots & h_{1, M, i} \\
\vdots & \ddots & \vdots \\
h_{N, 1, i} & \cdots & h_{N, M, i}
\end{array}\right]
$$

The elements of $H_{i}$ are assumed to be single tap channel (i.e. no inter-symbol interference exists) and they contain two Gaussian parts, real and imaginary. The total channel is then:

$$
H=\left[H_{1}^{T} H_{2}^{T} \cdots H_{K}^{T}\right]^{T}
$$


and the leakage channel is given by:

$$
\hat{H}=\left[H_{1}^{T} \cdots H_{i-1}^{T} H_{i+1}^{T} \cdots H_{K}^{T}\right]^{T}
$$

The transmitted vector from the base station $\mathrm{X}$ is the sum of the transmitted vector for all of the users:

$$
X=\sum_{i=1}^{K} w_{i} s_{i}
$$

where $w_{i} \in \mathrm{C}^{\mathrm{N} \times \mathrm{M}}$ is the beamforming vector for user $i$ and $s_{i}$ is the data symbol for that user. The received signal for user $i$ is:

$$
y_{i}=H_{i} X+n_{i}
$$

where $n_{i}$ is the noise vector at user $i$ with variance equal to $\sigma^{2}$.

The system capacity is given by the equation (in units of $b / s / H z$ ):

$$
\begin{gathered}
C=\log _{2}(1+\operatorname{SINR}) \\
C=\log _{2}\left(1+\frac{S}{I+N_{0}}\right)
\end{gathered}
$$

The signal power received by the user is $\left|H_{i} w_{i}\right|$, with interfering signal $\sum_{\substack{k=1 \\ k \neq i}}^{K}\left|H_{i} w_{k}\right|$, so that equation (7) can be written:

$$
C=\log _{2}\left(1+\frac{\left|H_{i} w_{i}\right|}{\sum_{\substack{k=1 \\ k \neq i}}^{K}\left|H_{i} w_{k}\right|+N \sigma_{i}^{2}}\right)
$$

\section{SLNR Optimization}

The aim is to find a precoder which maximizes the signal to leakage and noise ratio (SLNR): in other words which increases the power through the channel to the intended user while simultaneously minimizing the interference to other users.

As stated in section 1 above, the SINR leads to a coupled optimization problem which is solved by extending the SLNR as in [10-12]. The SLNR is given by:

$$
S L N R_{i}=\frac{S}{L+N_{0}}
$$

where the leakage term is:

$$
L=\sum_{\substack{k=1 \\ k \neq i}}^{K}\left|H_{k} w_{i}\right|
$$

The problem may be formulated in two ways. The first ignores the noise term and maximizes the signal to leakage ratio: 


$$
\frac{S}{L}=\frac{\left|H_{i} w_{i}\right|}{\sum_{\substack{k=1 \\ k \neq i}}^{K}\left|H_{i} w_{k}\right|}
$$

This equation can be rewritten thus:

with solution [9]:

$$
\frac{S}{L}=\frac{w_{i}^{H} H_{i}^{H} H_{i} w_{i}}{w_{i}^{H} \hat{H}_{i}^{H} \hat{H}_{i} w_{i}}
$$

$$
\frac{w_{i}^{H} H_{i}^{H} H_{i} w_{i}}{w_{i}^{H} \hat{H}_{i}^{H} \hat{H}_{i} w_{i}} \leq \lambda_{\max }\left(H_{i}^{H} H_{i}, \hat{H}_{i}^{H} \hat{H}_{i}\right)
$$

where $\lambda_{\max }$ is the largest eigenvalue. The optimal beamformer is:

$$
w_{i}^{o} \propto \max . G E V\left(H_{i}^{H} H_{i}, \hat{H}_{i}^{H} \hat{H}_{i}\right)
$$

and if $\hat{H}_{i}^{H}$ is invertible then (14) will be:

$$
w_{i}^{o} \propto \max . E V\left(\left(\hat{H}_{i}^{H} \hat{H}_{i}\right)^{-1} H_{i}^{H} H_{i}\right)
$$

The method is extended to include the effect of noise [11], so (11) becomes:

$$
\frac{S}{L+N_{0}}=\frac{\left|H_{i} w_{i}\right|}{\sum_{\substack{k=1 \\ k \neq i}}^{K}\left|H_{i} w_{k}\right|+N \sigma_{i}^{2}}
$$

The corresponding equations for (14) and (15) are:

$$
\begin{array}{r}
w_{i}^{o} \propto \max . G E V\left(H_{i}^{H} H_{i}, \hat{H}_{i}^{H} \hat{H}_{i}+N \sigma_{i}^{2} I\right) \\
w_{i}^{o} \propto \max \cdot E V\left(\left(\hat{H}_{i}^{H} \hat{H}_{i}+N \sigma_{i}^{2} I\right)^{-1} H_{i}^{H} H_{i}\right)
\end{array}
$$

\section{Performance of Eigenvalue Decomposition and Generalized Eigenvalue Decomposition: EVD and GEVD}

The SLR and SLNR approach has been proposed previously [10-12], but a new viewpoint is obtained here by applying the method over a wider range and analyzing the effects on the behavior of the EVD and GEVD, permitting one to understand the overall benefits for the total system resulting from SLNR maximization. The GEVD for two matrices $A$ and $B$ is given by

$$
A v=B \lambda v
$$

If $B$ is not singular (i.e. $B^{-1}$ exists) then we can say

$$
B^{-1} A v=\lambda v
$$

which is the same as $D v=\lambda v$ for $D=B^{-1} A$. In general if we have a matrix $c \in \mathbb{C}^{N \times M}$ where $N=N_{1}+N_{2}$, we can say it is composed from two matrices $a$ and $b$ :

$$
c=\left[\begin{array}{ll}
a^{T} & b^{T}
\end{array}\right]^{T}
$$

The GEVD for the two matrices $A=a^{H} \times a$ and $B=b^{H} \times b$ gives two matrices $\lambda$ 
and $v$. The columns of matrix $v$ contain candidates to be in the null space of matrix $b$, i.e. give zeros when multiplied by the matrix $b$. If $a$ is the user channel, $b$ is the leakage channel and $c$ is the aggregated channel, then $v$ is expected to have at least one vector that gives a zero and a non-zero result when multiplied by $b$ and $a$ respectively. However, that can be misleading as this assumption depends on the dimension of $c$. Table I compares the GEVD for different cases of $M, N, N_{1}$ and $N_{2}$.

Table I

\begin{tabular}{c|c|c|c}
\multirow{2}{*}{ Case } & Sub Case & $\begin{array}{c}\text { Polution(s) } \\
\text { exist? }\end{array}$ & $\begin{array}{c}\text { Number } \\
\text { of solutions }\end{array}$ \\
\hline$N<M$ & & yes & $>N_{1}$ \\
\hline$N=M$ & $N_{1}=N_{2}$ & yes & $=N_{1}$ \\
\hline$N>M$ & $N_{2} \geq M$ & no & \\
\hline & $N_{2}<M$ & yes & $\left(M-N_{2}\right)$
\end{tabular}

Note that the solution vector is associated with the largest eigenvalue for this case. If the equation is flipped to be $\operatorname{GEVD}(B, A)$ then the eigenvector associated with the lowest absolute value should be selected.

\section{$5 \quad$ Results}

In this section a set of carefully selected representative results is presented to give a clear understanding of the behavior of the system in terms of the SLNR criterion.

Figure 2 below shows performance versus increasing SNR for different numbers of users. As can be seen, the increase in number of users per system increases the capacity due to the increment in total data transferred through the wireless channel. 


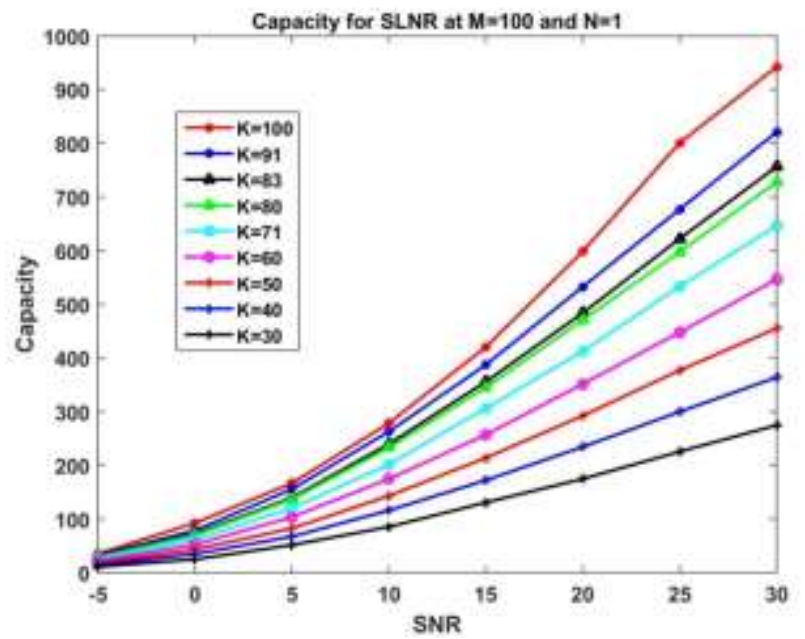

Figure 2: System performance in terms of capacity versus SNR for different numbers of single antenna users.

The monotonicity of the curve does not hold in all cases; as seen in Figure 3 the number of antennas per user affects the performance. For low numbers of users, the curves retain the same behavior, but with increasing numbers of users the curves take another shape. Although the shapes are different and there are two sets of curves, the behavior at each SNR is the same. The capacity starts at a certain level and increases to a maximum after which it decreases. At $5 \mathrm{~dB}$ SNR the peak is for 60 users while for $10 \mathrm{~dB}$ the peak falls to 50 users. At higher SNR the higher capacity relation still holds.

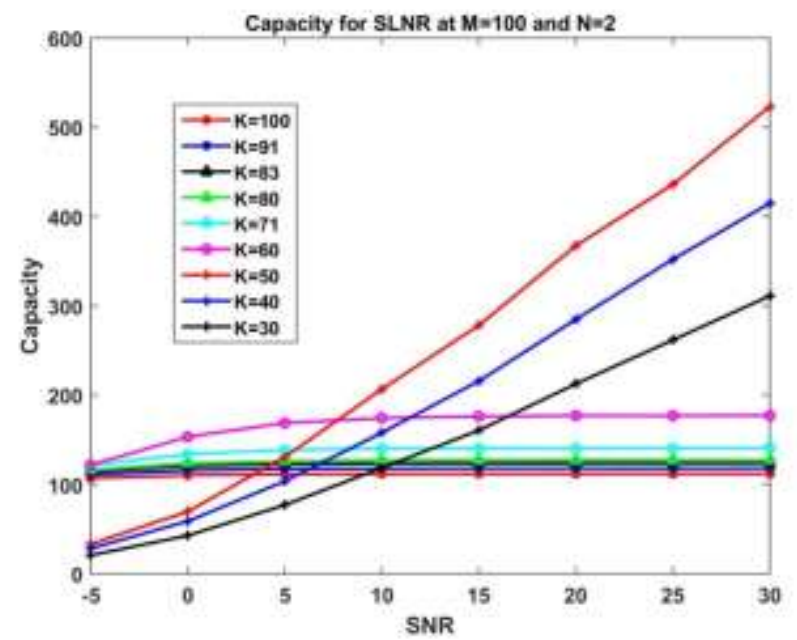

Figure 3: System performance in terms of capacity versus SNR for different numbers of two antenna users 
As can be seen from the figures above, the capacity due to the increment in number of users served by the system can be affected by the number of antennas per user. To give more clarification, Figure 4 illustrates the effect of varying numbers of user antennas per scenario. There are two types of curves. The first set, for fewer user antennas, resembles the performance shown in Figure 3, also showing increasing capacity with increasing antenna numbers. The second set has a different shape, tending to saturate at lower SNR values. Below $5 \mathrm{~dB}$ SNR the peak is at 4 antennas per user while after $10 \mathrm{~dB}$ SNR the peak moves to 3 antennas per user for 30 users served by a 100 antenna base station.

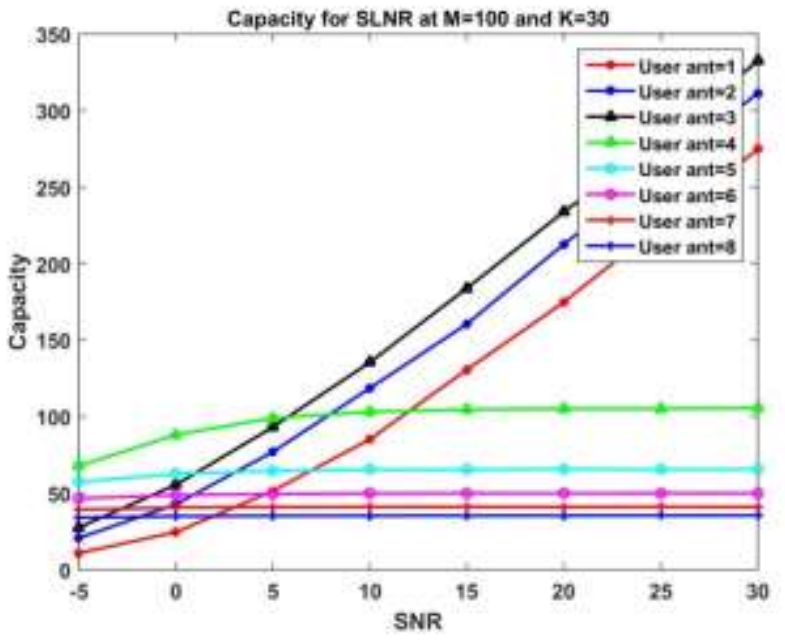

Figure 4: System performance in terms of capacity versus SNR for different numbers of antennas per users.

Another perspective can be got by combining two criteria (the number of users and the number of antennas at the base station) as in Figure 5. This figure shows that the curves have peaks, at different positions and different values. The position of the peak (in terms of number of users) tends to move down with increase of the ratio $\mathrm{M} / \mathrm{K}$ as we increase the number of antennas per user. 


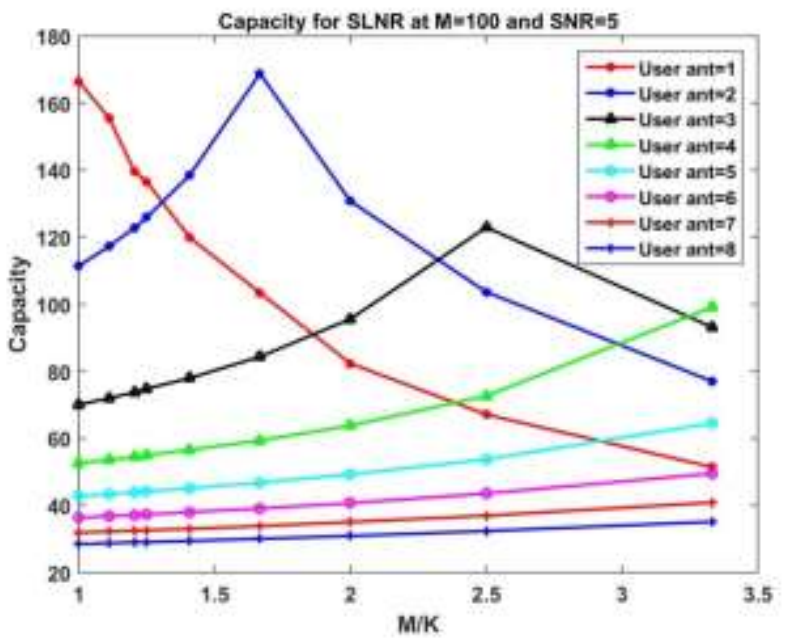

Figure 5: System performance in terms of capacity versus base station antennas to users ratio.

Another perspective to the problem can be seen in Figure 6. Here we see a peak also in terms of antenna users: at 2 antennas per user for higher number of users (60 and more), with the peak at higher numbers of antennas as the number of users decreases. The capacity has a concave shape over number of users and it occurs at 60 users when the number of base station antennas is 100 and the SNR is $0 \mathrm{~dB}$.

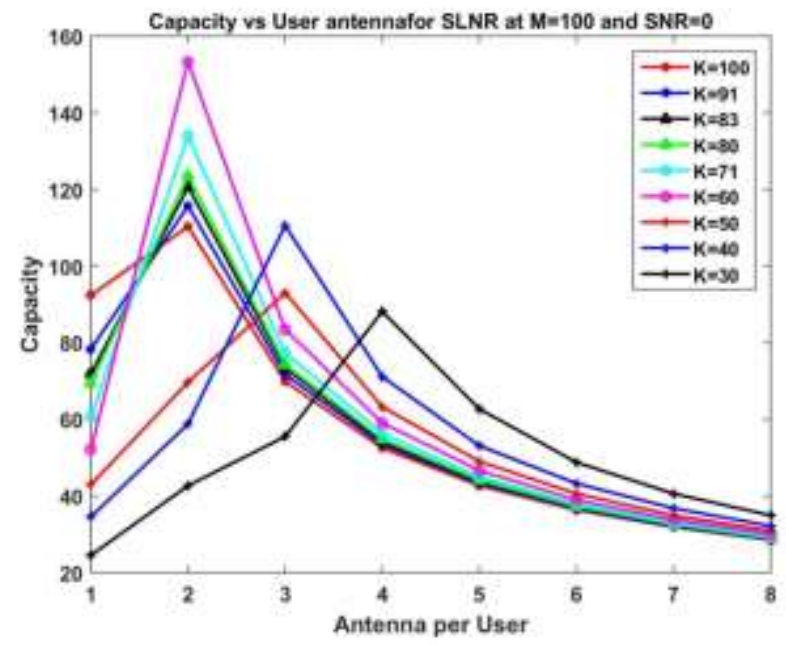

Figure 6: System performance in terms of capacity versus antenna per user for different numbers of users per cell. 


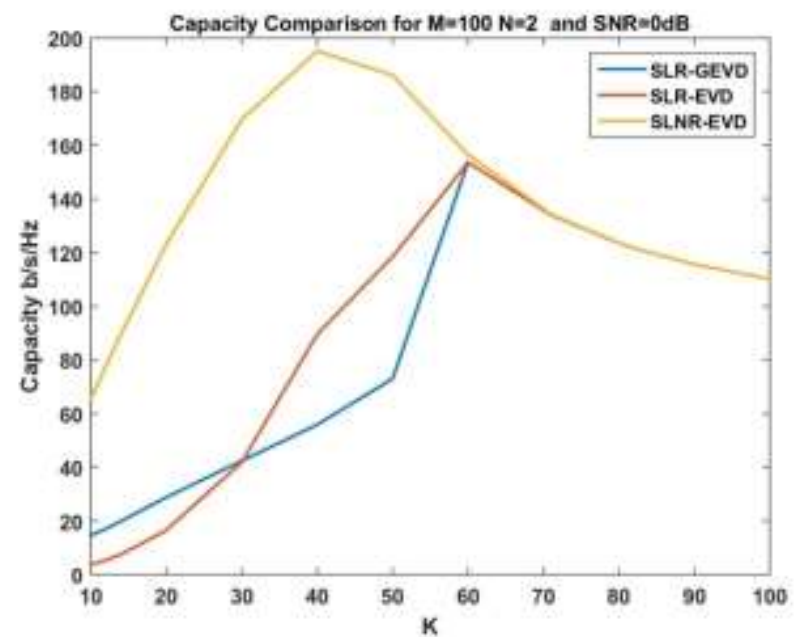

Figure 7: Capacity versus number of users, comparing three methods.

Finally, Figure 7 shows a comparison three approaches. The first variation uses the eigenvalue decomposition (in blue) instead of the generalized eigenvalue decomposition (orange line), i.e. using equation (15) instead of (14). The third variation (yellow line), shows results where the effect of noise was included using equation (18) to evaluate the weights of the beamformer.

\section{Conclusion}

The performance of SLR based on beamforming with MU-MIMO is presented. The resulting system is not monotonic in all of the four dimensions of interest, namely numbers of base station antenna, SNR, numbers of user antennas and the number of users served by the base station. It is shown that there are peak in performance, and that the capacity variation follows concave curves with variation of number of users and number of handset antennas.

\section{REFERENCES}

[1] A. Alexiou and M. Haardt, "Smart antenna technologies for future wireless systems: trends and challenges," IEEE communications Magazine, vol. 42, pp. 90-97, 2004.

[2] Q. H. Spencer, C. B. Peel, A. L. Swindlehurst, and M. Haardt, "An introduction to the multi-user MIMO downlink," IEEE communications Magazine, vol. 42, pp. 60-67, 2004.

[3] D. Gesbert, S. Hanly, H. Huang, S. S. Shitz, O. Simeone, and W. Yu, "Multi-cell MIMO cooperative networks: A new look at interference," IEEE Journal on Selected Areas in Communications, vol. 28, pp. 1380-1408, 2010.

[4] Q. H. Spencer, A. L. Swindlehurst, and M. Haardt, "Zero-forcing methods for downlink spatial multiplexing in multiuser MIMO channels," IEEE Transactions on Signal Processing, vol. 52, pp. 461-471, 2004. 
[5] C. B. Peel, B. M. Hochwald, and A. L. Swindlehurst, "A vector-perturbation technique for near-capacity multiantenna multiuser communication-part I: channel inversion and regularization," IEEE Transactions on Communications, vol. 53, pp. 195-202, 2005.

[6] F. Zhang, Y. Huang, S. Jin, L. Jiang, and G. Wang, "Reduced-backhaul coordinated beamforming for massive MIMO heterogeneous networks," in 2015 IEEE Wireless Communications and Networking Conference (WCNC), 2015, pp. 129-134.

[7] F. Rashid-Farrokhi, K. R. Liu, and L. Tassiulas, "Transmit beamforming and power control for cellular wireless systems," IEEE Journal on Selected Areas in Communications, vol. 16, pp. 1437-1450, 1998.

[8] W. Yu and T. Lan, "Transmitter optimization for the multi-antenna downlink with per-antenna power constraints," IEEE Transactions on Signal Processing, vol. 55, pp. 2646-2660, 2007.

[9] S. Shi, M. Schubert, and H. Boche, "Rate optimization for multiuser MIMO systems with linear processing," IEEE Transactions on Signal Processing, vol. 56, pp. 40204030, 2008.

[10] A. Tarighat, M. Sadek, and A. H. Sayed, "A multi user beamforming scheme for downlink MIMO channels based on maximizing signal-to-leakage ratios," in Acoustics, Speech, and Signal Processing, 2005. Proceedings.(ICASSP'05). IEEE International Conference on, 2005, pp. iii/1129-iii/1132 Vol. 3.

[11] M. Sadek, A. Tarighat, and A. H. Sayed, "Active antenna selection in multiuser MIMO communications," IEEE Transactions on Signal Processing, vol. 55, pp. 1498-1510, 2007.

[12] M. Sadek, A. Tarighat, and A. H. Sayed, "A leakage-based precoding scheme for downlink multi-user MIMO channels," IEEE Transactions on Wireless Communications, vol. 6, pp. 1711-1721, 2007.

[13] M. Jung, Y. Kim, J. Lee, and S. Choi, "Optimal number of users in zero-forcing based multiuser MIMO systems with large number of antennas," Journal of Communications and Networks, vol. 15, pp. 362-369, 2013.

[14] K.W. Hameed, R.A. Abd-Alhameed and A. Radwan, "Optimal Array size for Multiuser MIMO," in IWCMC-SICM, Cyprus, 2018. 